\title{
REFERENCE
}

1. E. A. Coddington and N. Levinson, Theory of ordinary differential equations, McGraw-Hill, New York, 1955.

Louisiana State University

\section{LOWER BOUNDS FOR SOLUTIONS OF DIFFERENTIAL INEQUALITIES IN HILBERT SPACE}

\author{
HAJIMU OGAWA ${ }^{1}$
}

Let $A$ be an operator in a Hilbert space and let $u(t)$ be in the domain of $A$ for each $t \in[0, \infty)$. Assuming $u$ is strongly differentiable, $A u$ strongly continuous and $d u / d t$ strongly piecewise continuous, all with respect to $t$, we define

$$
L u=\frac{d u}{d t}-A u
$$

In the case where $A$ is symmetric, i.e., $(A u, v)=(u, A v)$, Cohen and Lees [1] obtained lower bounds for solutions of differential inequalities of the form

$$
|L u(t)| \leqq \phi(t)|u(t)| \text {. }
$$

They proved that if $\phi \in L_{p}(0, \infty)$ for some $p$ with $1 \leqq p \leqq 2$, then any solution of (2) such that $u(0) \neq 0$ satisfies

$$
|u(t)| \geqq K e^{\lambda t},
$$

where $K>0$ and $\lambda$ are constants depending on the solution. Assuming that $A$ is selfadjoint, Agmon and Nirenberg [2] found a simpler

Presented to the Society, April 13, 1965; received by the editors October 2, 1964.

${ }^{1}$ This work was supported by the Air Force Office of Scientific Research under the contract AFOSR 553-64. 
proof of this result, as well as some extensions, by means of convexity theorems. The purpose of this paper is to present still simpler proofs, assuming only that $A$ is symmetric, of the theorem of Cohen and Lees for $p=2$ and of the extensions of Agmon and Nirenberg.

Theorem. Suppose $A$ is symmetric and let $u$ be a solution of (2).

(i) If $\phi \in L_{p}(0, \infty)$ for some $p$ with $2 \leqq p \leqq \infty$, then

$$
|u(t)| \geqq|u(0)| \exp \left[\lambda t-\mu(t+1)^{2-2 / p}\right] .
$$

(ii) If $\phi(t) \leqq K(t+1)^{\alpha}, \alpha>0$, then

$$
|u(t)| \geqq|u(0)| \exp \left[\lambda t-\mu(t+1)^{2 \alpha+2}\right] .
$$

In each case, $\lambda$ is a constant depending on $u$, while $\mu$ is a constant depending only on $\phi$.

Proof. Assuming $|u(t)| \neq 0$ for all $t \geqq 0$, we first note from (1) and the hypothesis that $A$ is symmetric that

$$
\frac{d}{d t} \log |u|^{2}=\frac{2(A u, u)+2 \operatorname{Re}(L u, u)}{|u|^{2}} .
$$

Moreover, the strong differentiability of $u$ and the strong continuity of $A u$ imply

$$
\frac{d}{d t}(A u, u)=2 \operatorname{Re}\left(A u, \frac{d u}{d t}\right)
$$

It follows that

$$
\begin{aligned}
|u|^{4} \frac{d}{d t} \frac{(A u, u)}{|u|^{2}}= & 2|u|^{2} \operatorname{Re}(A u, A u+L u) \\
& -2(A u, u) \operatorname{Re}(A u+L u, u) \\
= & 2\left|A u+\frac{1}{2} L u\right|^{2}|u|^{2}-\frac{1}{2}|L u|^{2}|u|^{2} \\
& -2\left[\operatorname{Re}\left(A u+\frac{1}{2} L u, u\right)\right]^{2}+\frac{1}{2}[\operatorname{Re}(L u, u)]^{2} .
\end{aligned}
$$

Applying Schwarz's inequality and (2) to this equation, we then find

$$
\frac{d}{d t} \frac{(A u, u)}{|u|^{2}} \geqq-\frac{1}{2} \phi^{2} .
$$

For the case (i), we assert that

$$
\frac{(A u, u)}{|u|^{2}} \geqq \lambda-M t^{1-2 / p},
$$

where $\lambda=(A u(0), u(0)) /|u(0)|^{2}$ and $M$ is a constant depending only 
on $\phi$. For $p=2$ and $p=\infty$, this is an immediate consequence of (6) For $2<p<\infty$, we make use of Hölder's inequality to obtain the estimate

$$
\int_{0}^{t} \phi^{2} d s \leqq\left(\int_{0}^{t} \phi^{p} d s\right)^{2 / p}\left(\int_{0}^{t} d s\right)^{1-2 / p} \leqq M t^{1-2 / p},
$$

so (7) follows from the integration of (6).

Applying (7) and (2) to the equation (5), we see that

$$
\frac{d}{d t} \log |u(t)| \geqq \lambda-M t^{1-2 / p}-\phi(t) .
$$

Integrating this inequality and applying Hölder's inequality to the term in $\phi$, we find that

$$
\log |u(t)| \geqq \log |u(0)|+\lambda t-\frac{p}{2 p-2} M t^{2-2 / p}-N t^{1-1 / p}
$$

where $N$ depends only on $\phi$. Since the last two terms are bounded below by

$$
-\mu(t+1)^{2-2 / p}
$$

for some constant $\mu,(3)$ follows.

For the case (ii), the lower bound (4) is easily verified if we integrate (6) and apply the resulting inequality, together with (2), to the equation (5).

If $|u(0)| \neq 0$, the assumption that $|u(t)| \neq 0$ for $t>0$ can easily be shown to be valid. For suppose the contrary, and let $t_{0}$ be the first point where $|u(t)|=0$. Then (3) or (4) holds for $0 \leqq t<t_{0}$, and by continuity the bound also holds at $t_{0}$, thus contradicting $\left|u\left(t_{0}\right)\right|=0$.

\section{BIBLIOGRAPHY}

1. P. J. Cohen and M. Lees, Asymptotic decay of solutions of differential inequalities, Pacific J. Math. 11 (1961), 1235-1249.

2. S. Agmon and L. Nirenberg, Properties of solutions of ordinary differential equations in Banach space, Comm. Pure Appl. Math. 16 (1963), 121-239.

UNIVERSITY OF CALIFORNIA, RIVERSIDE 